\title{
EL «FACTOR HUMANO»Y LA DISTRIBUCIÓN SEXUAL DEL TRABAJO EN EL DISCURSO DE LA ORGANIZACIÓN CIENTÍFICA DEL TRABAJO (ESPAÑA, 1922-36)"
}

\author{
José Martínez Pérez
}

Universidad de Castilla-La Mancha

\section{RESUMEN}

A lo largo del primer tercio del siglo XX, y especialmente a partir de la década de los años veinte, se empezó a difundir en España un discurso sobre la higiene y seguridad en el trabajo que, aun sin rechazar el valor de la actuación sobre el medio ambiente fabril, hacía especial hincapié en la necesidad de tomar en cuenta lo que denominaban como «factor humano». Impulsado fundamentalmente por los incipientes médicos y psicólogos del trabajo, este discurso quedó enmarcado tanto en las corrientes de pensamiento biológico de la patología constitucional, como en las ideas económicas de la llamada Organización Científica del Trabajo, y planteó la necesidad de tomar en cuanta las características somáticas y psíquicas de las personas para llevar a cabo una distribución «racional» de las mismas en el mercado de trabajo. El artículo intenta poner de relieve cómo dicho discurso contenía elementos capaces de servir para la atribución de roles específicos dentro de la actividad laboral en función de los características biológicas y psicológicas de hombres y mujeres, facilitando a su vez de esta manera la legitimación de una distribución sexual del trabajo que contribuía a reforzar la organización social de género de la época.

PALABRAS CLAVE: Género y Medicina, Salud e Higiene en el Trabajo, Psicología del Trabajo, Siglo XX, España.

MEDICINE AND THE SEXUAL DISTRIBUTION OF WORK: AN ANALYSIS OF THE SCIENTIFIC DISCOURSE ON THE ROLE OF THE «HUMAN FACTOR» IN IMPROVING PERFORMANCE AT WORK (SPAIN 1922-1936)

* Este trabajo ha sido elaborado con cargo al proyecto de referencia PAI-05-009 de la Consejería de Sanidad de la Junta de Comunidades de Castilla-La Mancha. Para su elaboración se ha contado también con una ayuda de la Fundación para la Investigación Sanitaria en Castilla-La Mancha (No Ref.: GCS-2006_E/04). 
JOSÉ MARTÍNEZ PÉREZ

\begin{abstract}
During the first third of the twentieth century, and especially after the 1920s, a discourse on occupational hygiene and safety began to develop in Spain. This discourse, without rejecting the value of the work carried out in the factory environment, particularly stressed the need to take into account what was called the «human factor». Promoted mainly by the budding occupational doctors and psychologists, this discourse became part of both the lines of biological thinking of constitutional pathology as well as the economic ideas of the so-called OCT, and it expounded the need to take the somatic and psychic characteristics of people into account in order to carry out a «rational» distribution of the same in the workplace. The article aims to highlight the way in which this discourse contained elements that would help to attribute specific roles within the workplace based on the biological and psychological characteristics of men and women, so facilitating the legitimisation of a sexual distribution of work which helped to reinforce the social organisation of gender at that time.
\end{abstract}

KEY WORDS: Gender and medicine, Occupational health and safety, Occupational psychology, Twenty Century, Spain.

\title{
INTRODUCCIÓN
}

A finales del siglo XIX se desplegó una importante actividad legislativa encaminada a regular diversos aspectos relacionados con la siniestralidad labora ${ }^{1}$. En el caso de España, esta actuación normativa tuvo como uno de sus hitos iniciales la Ley de Accidentes del Trabajo de 30 de enero 1900². Con ella se ponía plenamente de manifiesto el interés del Estado por tratar de hacer frente a una cuestión que se estaba convirtiendo en un problema para la nación por sus repercusiones a nivel individual y, muy especialmente, colectivo $^{3}$. En efecto, este proceso normativo, que estuvo muy centrado desde el principio en el auxilio a la invalidez que provocaban los accidentes ${ }^{4}$, no perseguía sólo combatir las penosas consecuencias que la siniestralidad laboral comportaba para los obreros y sus familias; esto es, mitigar el daño económico y humano que en ellos provocaba la muerte o la incapacidad a que daba lugar. Con ese conjunto de leyes se pretendía también hacer frente a las negativas repercusiones que los accidentes del trabajo ejercían sobre algo que se

1 Soto Carmona, A. (1985), «La higiene, la seguridad y los accidentes del trabajo. España (1874-1936)», Civitas. Revista Española de Derecho del Trabajo, 23, 389-423, p. 392.

2 Su Reglamento es de 28 de julio de ese año. Soto CARMONA (1985), pp. 393-394.

3 RodRíGUEZ-OCAÑA, E. (1993), «Industrielle Gesundheitsgefährdung und Medizin in Spanien, 1850-1936. Eine Annäherung an den medizinischen Diskurs». En MiLleS, D. (ed.), Gesundheitsrisiken, Industriegesellschaft und soziale Sicherungen in der Geschichte, Bremerhaven, Wirtschaftsverlag NW, Verlag für neue Wissenschaft GmbH, 419-440, pp. 424-425.

4 Soto Carmona (1985), p. 393; y p. 391. 
percibía como un elemento fundamental para la buena marcha de la nación: el mantenimiento de la producción.

Y es que, desde finales del siglo XIX, la necesidad de potenciar la seguridad se relacionó con la idea de que, al disminuir los riesgos para la integridad física de los obreros, se estaría favoreciendo su capacidad productiva ${ }^{5}$. De hecho, la noción de que resultaba conveniente mantener al obrero en buenas condiciones físicas y morales para hacerlo más productivo formaba parte del entramado ideológico del «paternalismo industrial», un programa de actuaciones que se proponía en ese período para conseguir un trabajador más capaz de cumplir con las tareas que de él se esperaban y en el que la Medicina tenía mucho que ofrecer6.

No obstante, a finales del siglo XIX y comienzos del siglo XX esta «disciplina industrial», este conjunto de técnicas destinado a atraer a la fábrica al trabajador y a adaptarlo a la actividad productiva ${ }^{7}$, se estaba mostrando incapaz, al menos en la que se ha denominado como su variedad «patriarcal» ${ }^{8}$, de cumplir satisfactoriamente con sus objetivos en el marco de las transformaciones estructurales del capitalismo que se vieron aceleradas en esos años ${ }^{9}$. Nuevos sistemas se estaban ofreciendo como alternativa al paternalismo industrial y pronto iban a sustituirlo en América y Europa.

Uno de ellos, que se dio en llamar «Organización Científica del Trabajo», comenzó a extenderse en España tras la Primera Guerra Mundial. Tras haber sido presentado inicialmente como un medio eficaz para mejorar la producción en el estricto plano de las empresas, fue siendo considerado posterior-

5 Ese era, por ejemplo, el punto de vista que sostenían los ingenieros. RODRíGUEZ OCAÑA (1992), «Paz, trabajo, higiene. Los enunciados acerca de la Higiene Industrial en la España del siglo XIX». En HuERTAS, R.; CAMPOS, R. (eds.), Medicina social y clase obrera en Espa$\tilde{n} a$ (siglos XIX y XX) (2 vols.), vol. 2, Madrid, Fundación de Investigaciones Marxistas, 383406, p. 393.

6 Sierra Álvarez, J. (1990), El obrero soñado. Ensayo sobre el paternalismo industrial (Asturias, 1860-1917), Madrid, Siglo XXI, p. 80. Las propuestas de los higienistas españoles de las tres últimas décadas del siglo XIX habrían servido para alentar el paternalismo empresarial, según RODRÍGUEZ OCAÑA (1992), p. 395.

7 Sierra Álvarez (1990), pp. 14-15.

8 Se ha distinguido entre una forma de paternalismo «patriarcal» y otra «liberal», que se caracterizarían respectivamente, entre otros rasgos, por el grado en que el funcionamiento de los aparatos institucionales promovidos por los patronos en torno a sus fábricas se decantaban hacia alguno de los dos principios activos que figuraban en él: la asistencia y la previsión. SiERRA ÁlVAREZ (1990), pp. 146-149.

9 Una descripción de esas transformaciones puede verse en SiERrA Álvarez (1990), pp.158-153. 
mente como portador de métodos susceptibles de ser aprovechados en la administración de la nación. Entre ellos, ocupaban un lugar destacado los que iban dirigidos al estudio y gestión del «factor humano». No debe extrañar, por tanto, que el abordaje de las cuestiones relacionadas con la salud y la enfermedad, como era el caso de la siniestralidad laboral, pasaran a ocupar un lugar destacado en el conjunto de medidas que la Organización Científica del Trabajo (OCT, en adelante) proponía para modernizar España.

El presente trabajo tiene como objetivo mostrar los rasgos más sobresalientes de dicho programa y, más concretamente, aquellos aspectos del mismo que lo convirtieron en impulsor de ese discurso de género cuya presencia es posible discernir de manera conspicua en la producción científica y médica occidental $^{10}$. Para ello, trataré de mostrar, en primer lugar, los rasgos principales de la OCT. Posteriormente, me ocuparé de exponer la forma en que sus contenidos se pusieron en marcha en la España anterior a la Guerra Civil y alentaron la formación de un discurso que propiciaba la legitimación de una distribución sexual del trabajo.

\section{LA OCT Y EL ESTUDIO DEL «FACTOR HUMANO»}

El programa de la OCT perseguía, de acuerdo con «métodos rigurosamente científicos», la «disposición de los diferentes factores de la producción (...) que conducen al óptimo rendimiento ${ }^{11}$. Dado que el trabajador fue considerado desde el primer momento como uno de los elementos más importantes para determinar los resultados de la actividad laboral, se empezaron a desarrollar ya en el siglo XIX estudios científicos sobre el «motor humano» con

10 Véase al respecto: Medina, R. (1999), «Ideas para perder la inocencia sobre los textos de ciencia». En BARRAL, M. J. et al. (eds.), Interacciones ciencia y género. Discursos y prácticas científicas de mujeres, Barcelona, Icaria, pp. 103-128; SÁNCHEZ, D. (1999), «Androcentrismo en la ciencia. Una perspectiva desde el análisis crítico del discurso». En BARRAL et al. (eds.), pp. 161-184. Un valioso examen sobre la producción historiográfica en torno a la construcción histórica de los discursos de género aparece recogido en ORTIZ, T. (2006), Medicina, historia y género. 130 años de investigación feminista, Oviedo, KRK, 159-206.

11 Cfr. Palacios, G. (1928-1929a), «Reseña de 'Organización Científica del Trabajo.- I. Las ideas', por César de Madariaga. Biblioteca Marvá, Madrid», Revista de Organización Científica, 1, 57-59, p. 58. César de Madariaga fue Director Técnico del Instituto de Orientación y Selección Profesional y Director General de Comercio. BACHILlER BAEZA, A. (1985), La medicina social en España (El Instituto de Reeducación y la Clínica del Trabajo 19221937), Valladolid, Secretariado de Publicaciones de la Universidad de Valladolid, p. 12. 
vistas a resolver los problemas del trabajo mediante una perspectiva que se proclamaba neutral y objetiva. Como ha indicado Rabinbach, los expertos en fatiga, nutrición y fisiología sometieron el cuerpo a una detallada investigación en el laboratorio. De esta tarea surgió una nueva disciplina -la «Ciencia Europea del Trabajo» (CET, en adelante) ${ }^{12}$ - que se convirtió en una herramienta intelectual que los reformadores utilizaron para ayudarse en la labor de mediación en los conflictos entre trabajadores y empresarios. Esto brindó, a quienes se presentaban como expertos en esa disciplina, la oportunidad para reclamar una creciente cooperación con el Estado en cuestiones tan relevantes como la duración de la jornada laboral, el día de descanso semanal, los accidentes del trabajo, el seguro de enfermedad, los métodos más adecuados de formación, y el papel de las mujeres en la fuerza de trabajo ${ }^{13}$.

Aunque en 1913 era posible encontrar informes oficiales que reconocían el significado de la CET para la política pública, los años que precedieron a la Primera Guerra Mundial supusieron para la misma un momento de crisis. En ese período las ideas de Frederick Winslow Taylor sobre la organización industrial se empezaban a difundir por Europa con un impacto considerable ${ }^{14}$. El taylorismo presentaba indudables puntos de convergencia con la CET, lo que provocó que durante un tiempo rivalizaran entre sí. Los dos sistemas se presentaban como ciencias del trabajo y cada uno reivindicaba su capacidad para: analizar detalladamente las tareas del obrero y sus movimientos; mejorar la productividad y la eficiencia; y por último, trascender los intereses de clase y la ideología en beneficio de una organización económica racional y científica de la actividad laboral. Además, lo que es muy relevante de cara a los propósitos de este artículo, para ambos sistemas el cuerpo del trabajador era lo que constituía el centro de debate en el ámbito industrial. De este modo, como ha señalado Rabinbach, la racionalización de la producción se predicaba sobre la racionalización del cuerpo ${ }^{15}$.

La Primera Guerra Mundial puso de relieve la utilidad, tanto de la CET como del taylorismo, para incrementar la producción industrial de las nacio-

12 RABINBACH, A. (1992), The human motor. Energy, fatigue, and the origins of modernity, Berkeley-Los Angeles, University of California Press Rabinbach, 1992, p. 6.

13 RABINBACH (1992), pp. 208-10.

14 Sobre la figura de Taylor y su obra puede verse: LAYTON, E. (1975), «Taylor, Frederick Winslow». En GILLISPIE, C.C. (ed.), Dictionary of Scientific Biography, New York, Charles Scribner's Sons, 13, 271-272. Sobre la aplicación de sus ideas véase CORIAT, B. (1993), El taller y el cronómetro. Ensayo sobre el taylorismo, el fordismo y la producción en masa, Madrid, Siglo XXI.

15 RABINBACH (1992), pp. 238-9. 
nes en conflicto y combatir la fatiga de los trabajadores y de los soldados. Ello sirvió, no sólo para facilitar la aceptación de las nuevas ideas que transmitían ambas disciplina sobre la organización de la actividad industrial ${ }^{16}$, sino también, para acelerar la aproximación entre ellas. De hecho, la organización científica del trabajo de post-guerra fue una síntesis de ambos sistemas ${ }^{17}$. En efecto, la guerra creó una «atmósfera propicia» que habría servido para abrir una nueva fase en el desarrollo de la disciplina. Esta etapa, que se denominó «de extensión», habría servido para propiciar esa unión entre «la psicofisiología y el taylorismo» que sería «el origen de la verdadera organización científica del trabajo» ${ }^{18}$.

El sistema que fue configurándose a partir de 1918 mostraba dos rasgos destacados: la voluntad de elevar sus aportaciones más allá de la economía privada de la fábrica, contribuyendo ahora a la organización económica a nivel nacional e incluso internacional; y el afán por incluir de forma relevante entre sus herramientas a la fisiología, la psicotecnia, los test de aptitud, y la orientación profesional ${ }^{19}$. Mediante ellas, como veremos a continuación, la OCT se reveló como una disciplina cargada de un poderoso discurso de género que fomentaba una distribución de la actividad laboral en la que el sexo de los individuos constituía un factor relevante.

\section{SOBRE EL SEXO Y LAS APTITUDES DEL «FACTOR HUMANO» PARA EL TRABAJO}

España no se mantuvo ajena a esta tendencia que se ponía de manifiesto a nivel continental ${ }^{20}$. Una importante expresión de ello lo iba a ser la forma en que el Instituto de Reeducación Profesional de Inválidos del Trabajo (IRPIT, en adelante), creado como consecuencia de la aplicación de la Ley de Acci-

16 RABINBACH (1992), p. 271.

17 RABINBACH (1992), p. 273.

18 Palacios, G. (1928-1929b), «Reseña de 'La Organización Científica del Trabajo en Europa', por Paul Devinat. (...) Edic. Aguilar. Madrid, 1928», Revista de Organización Cientifica, 1 (3), 65-68, p. 66.

19 RABINBACH (1992), p. 275.

20 Tomas, J. R.; EstiviLl, J. (1979), «Apuntes para una historia de la organización del trabajo en España, 1900-1936», Sociología del Trabajo, 1, 17-43, 27-32; Soto CARMONA, A. (1989), El trabajo industrial en la España contemporánea (1874-1936), Barcelona, Anthropos, pp. 207-246; y HerRero, F.; CARPINTERO, H. (1999), «El taylorismo en España. Su divulgación durante el primer tercio del siglo XX», Revista de Historia de la Psicología, 20 (3-4), 307-314. 
dentes del Trabajo de 1922, planteó el modo de responder a los objetivos para los que fue creado ${ }^{21}$. Como veremos a continuación, su programa de actuaciones, no sólo incorporó buena parte de los postulados de la OCT, sino que, a través de ello, impulsó un discurso que servía para legitimar una distribución social del trabajo en la que el sexo de los ciudadanos representaba un factor fundamental.

Al IRPIT le fueron encomendadas las tareas de readaptación funcional de aquellos obreros que la hubieran perdido a consecuencia de un accidente laboral, su reeducación profesional, y la tutela social de los mismos una vez que hubieran sido reeducados. De este modo, el IRPIT, y quienes en él desarrollaban su labor profesional, se veían inmersos en un programa que obligaba a integrar diversas disciplinas. Ingenieros, médicos, cirujanos, pedagogos y psicólogos, abrazaron los principios teóricos y las prácticas de la OCT para intentar responder satisfactoriamente a los objetivos del centro ${ }^{22}$. Ello iba a servir, no sólo para impulsar la introducción de la OCT en España ${ }^{23}$ o para estimular la constitución de algunas especialidades médicas -Medicina del Trabajo ${ }^{24}$,

21 Una visión de conjunto sobre el desarrollo del IRPIT puede seguirse en PALACIOS SÁNChEz, J. (s.a.), «Evolución histórica». En PAlacios SÁnchez, J. (ed.), Historia del C.P.E.E. de Reeducación de Inválidos. Antiguo INRI, Madrid, M.E.C.-C.P.E.E., pp. 50-90; y PALACIOS SÁNCHEZ, J. (1990), «La institución pionera de la rehabilitación en España», Boletín del Real Patronato de Prevención y Atención a Personas con Minusvalía , n 15, 1-34, 1990, pp. 8-16. Acerca de sus actividades véase: BACHILLER BAEZA (1985), pp. 9-40.

22 Sobre el valor de ese programa para consolidar un modelo «médico» de las discapacidades véase: MARTínEZ-PÉREZ, J.; PORRAS, M. I. (2006), «Hacia una nueva percepción social de las personas con discapacidades: legislación, medicina y los inválidos del trabajo en España (1900-1936), Dynamis, 26, 195-219; MARTínEZ-PÉREZ, J. (2006), «Una nueva tecnología contra la siniestralidad laboral: innovación, Medicina y accidentes del trabajo en España (1920-1936)». En PÉrez-Bustamante, J.A. et al. (eds.), Actas del IX Congreso de la Sociedad Española de Historia de las Ciencias y de las Técnicas (2 vols.), 1337-1360, Cádiz, SEHCYT.

23 MALlart, J. (1974), «Cincuentenario del originariamente llamado Instituto de Orientación y Selección Profesional», Revista de Psicología General y Aplicada, 29, 929-1008, p. 935. José Mallart (1897-1989) fue uno de los más significados impulsores de la orientación profesional en España. Sobre sus aportaciones puede consultarse: PÉREZ FERNÁNDEZ, F. (1999), «José Mallart y la Orientación Profesional. Apuntes para una Historia de la Psicología aplicada en España», Revista de Historia de la Psicología, 20 (3-4), 95-106; PÉREZ FERNÁNDEZ, F. (2000), «Contribuciones a la difusión de la Organización Científica del Trabajo en España. La aportación de José Mallart», Revista de Historia de la Psicología, 21 (2-3), 191-204.

24 BAChILler BAEZA, 1985; y MENÉNDEZ-NAVARro, A.; RodríGueZ-OcaÑA, E. (2003), «From 'accident medicine' to 'factory medicine': Spanish occupational medicine in the Twentieth Century». En GrIECO, A. et al. (eds.), Origins of Occupational Health Associations in the World, Amsterdam, Elsevier, 207-16. 
Traumatología y Ortopedia ${ }^{25}-$ y disciplinas relacionadas con la Pedagogía -Formación profesional- y la Psicología -Psicotecnia y Orientación y Selección profesional26-, sino que iba a facilitar también el desarrollo de un discurso que poseía elementos muy susceptibles de ser empleados como base para la legitimación de una distribución sexual del trabajo.

En efecto, la labor de «reeducación profesional de los inválidos» que se desarrollaba en el Instituto implicaba, además del tratamiento médico encaminado a reconstruir los tejidos y órganos dañados para devolver al accidentado las funciones que hubiera podido perder, establecer qué nuevo oficio podría desempeñar el trabajador con una discapacidad, convencerle de la oportunidad de que lo aprendiera y enseñárselo ${ }^{27}$. Por ello, en 1923 se creó en el IRPIT una sección destinada a seleccionar a las personas más susceptibles de ser reeducadas y «a distribuirlas científicamente por los diversos talleres y lugares de trabajo» ${ }^{28}$; esto es, a cultivar la orientación y la selección profesional.

De este modo, en el IRPIT se iba a desarrollar una disciplina que era fuertemente fomentada desde la $\mathrm{OCT}^{29}$ y que, como me interesa destacar por su relevancia para los fines de este artículo, intentaba discernir, a través del estudio de las características somáticas y psicológicas del «factor humano», la capacidad de los diferentes individuos para desarrollar un determinado traba-

25 MARTíneZ-PÉREZ, J. (2006), «El obrero recuperado: Medicina del Trabajo, Ortopedia y el impacto de la tecnología médica sobre la imagen social de las personas con discapacidades (España, 1922-1936)» História, Ciências, Saúde - Manguinhos, 13, 349-373. (Versión en inglés en: http://www.scielo.br/scielo.php?script=sci_issuetoc\&pid=0104-597020060002\&lng=en\&nrm=iso)

26 Encinas, M.; RosA, A. (1990), «El desarrollo institucional de la Psicología en España», Revista de Historia de la Psicología, 11, 73-121, pp. 84-87; y MARTíNEZ-PÉREZ, J. (1994), «La Organización Científica del Trabajo y las estrategias médicas de seguridad laboral en España (1922-1936)», Dynamis, 14, 131-158, 150-152; MALLART, (1974), p. 935.

27 Oller, A. (1924) «Sobre orientación profesional», Los progresos de la clínica, 27 (146), 145-170, pp. 155-168. Oller fue el primer Director Médico del Instituto. Sobre este autor véase: BACHILler BAEZA, A. (1984), Historia de la Medicina del Trabajo en España. La obra científica del Prof. Antonio Oller Martínez, Valladolid, Secretariado de Publicaciones de la Universidad de Valladolid.

28 «El Instituto de Reeducación Profesional y sus actividades» (1933), Medicina del Trabajo e Higiene Industrial, 3, 51-85, p. 52; MALlaRT, J. (1932a), El Instituto Psicotécnico de Madrid, Madrid, Asociación Española para el Progreso de la Ciencia-Huelves, p. 105.

29 MedinA, R.; RodrígueZ OcAÑA, E. (1992), «La Medicina en la Organización Científica del Trabajo. El Instituto de Orientación Profesional (Psicotécnico) de Barcelona (19171936)». En Huertas, R.; CAMPOS, R. (eds.), Medicina Social y clase obrera (Siglos XIX y $X X)$ (2 vols.), Madrid, Fundación de Investigaciones Marxistas, vol. 2, pp. 459-490 (ver pp. 462-463). 
jo. Esto iba a propiciar que, desde muy pronto, al amparo del IRPIT, se desarrollara un discurso que legitimaba una distribución de la actividad laboral en la que el sexo de las personas representaba un factor extraordinariamente relevante.

Una prueba conspicua de ello la encontramos en un trabajo firmado por Mercedes Rodrigo (1891-1982) ${ }^{30}$, una psicóloga del Instituto, en que se ocupaba de «la Orientación Profesional femenina» ${ }^{31}$. Su autora aspiraba a que con él «debería quedar demostrado que la mujer pueda ambicionar ocupar puestos reservados hasta ahora únicamente a los hombres; que la inteligencia

30 Graduada en la Escuela Superior de Maestras de Madrid, su ciudad natal, Mercedes Rodrigo se dedicó algunos años a la enseñanza en el Colegio Nacional de Sordomudos y Ciegos y Anormales y en el patronato Nacional de Anormales. Viajó por diferentes países europeos y, beneficiándose de una beca de la Junta para Ampliación de Estudios, recabó en el Instituto J.J. Rousseau de Ginebra en 1920, donde se especializó en Orientación Profesional. En él trabajó al lado de su director - E. Claparéde, considerado fundador de la Psicotecnia europea-, completando también su formación en Psicología colaborando con Piaget. Esto le sirvió para, de nuevo ya en España, integrarse en la Sección de Orientación Profesional del IRPIT, en el Instituto Médico-Pedagógico y, tras su creación en 1928, en el Instituto Psicotécnico de Madrid, que dirigió durante la Guerra Civil. Antes de finalizar ésta hubo de exiliarse $\mathrm{y}$, tras una breve estancia en Suiza, se trasladó a Colombia. Como en España, también aquí desarrolló una importante labor pionera en la institucionalización y desarrollo de la Psicología, dirigiendo, tras su creación en 1947, el Instituto de Psicología Aplicada de la Universidad Nacional. Su labor en Colombia se vio truncada por la obligación de iniciar en 1950 un segundo exilio, ahora en Puerto Rico. Allí residiría hasta su muerte, trabajando como profesora de educación en la Universidad y ejerciendo, hasta 1972, la consulta psicológica en una clínica privada de San Juan y en una institución dedicada a ofrecer apoyo médico y psicológico a los veteranos norteamericanos de guerra. Sobre esta autora pueden verse: ARDILA, R. (1988), «Mercedes Rodrigo (1891-1982)», Revista Latinoamericana de Psicología, 20, 429-434; Herrero, F. (1997), «La Escuela de Ginebra en la Psicología Aplicada Española: La figura de Mercedes Rodrigo», Revista de Historia de la Psicología, 18 (1-2), 139-150; Herrero, F. (2003), Mercedes Rodrigo: una pionera de la Psicología Aplicada en España y Colombia, Tesis doctoral de la Universidad Complutense de Madrid, Servicio de Publicaciones. (Disponible en: http://www.ucm.es/BUCM/tesis/19972000/H/2/H2095901.pdf).

31 Rodrigo, M. (1926a), «La Orientación Profesional Femenina», Memorias del Instituto de Reeducación Profesional de Inválidos del Trabajo, 3, 44-49, p. 44. Este artículo reproduce la conferencia impartida por Rodrigo en el IV Congreso de Estudios Vascos (Vitoria, 1926), dedicado al tema de la «Orientación Profesional», publicada asimismo en: RoDRIGO, M. (1927), «La Orientación Profesional Femenina». En IV Congreso de Estudios Vascos: recopilación de los trabajos de dicho Congreso, celebrado en Vitoria del 25 de julio al $1^{\circ}$ de agosto de 1926 acerca de temas de orientación y enseñanzas profesionales, 147-150, Donostia-San Sebastián, Eusko Ikaskuntza. Está accesible en: http://www.euskomedia.org/PDFAnlt/congresos/04147150.pdf. 
femenina no es inferior a la masculina; que el llamado sexo débil es capaz de afrontar situaciones difíciles y otra porción de cosas más, todas ellas favorables, claro está, a mis compañeras de sexo» ${ }^{32}$. Como trataré de poner de relieve a continuación, Mercedes Rodrigo establecía en este artículo una serie de argumentos que podían resultar contraproducentes para defender estos planteamientos. Desde mi punto de vista, al recurrir a ellos, la autora ahondaba y daba legitimidad, desde la pretendida objetividad de la disciplina que cultivaba, al establecimiento de una distribución del trabajo en la que el sexo de las personas había de constituirse en referente fundamental.

Desde el principio, Mercedes Rodrigo mostraba cómo el sexo constituía un factor relevante para llevar a cabo actuaciones encaminadas a intervenir de forma racional sobre la organización del mercado laboral. Para ella, la Orientación profesional podía hacerse de manera igual para los niños y las niñas sólo «hasta cierto punto». Según argumentaba, la razón de esta disparidad se debía a que, de los tres factores principales de toda orientación —el sujeto, la profesión y el mercado de trabajo - variaba «esencialmente el elemento sujeto». Esto le hacía sostener que las niñas «necesitan que les aconseje una mujer $\rangle^{33}$. La autora potenciaba de este modo la existencia de diferencias entre los dos $\operatorname{sexos}^{34}$, y lo remarcaba al indicar que, aunque «en la práctica de la orientación es posible admitir una orientadora para los muchachos», no sería «nunca» adecuado «un hombre para las niñas» debido a que se darían «muchas situaciones» en las que se plantearían «cuestiones de salud, de moral, o de asuntos familiares que son mucho más fáciles de tratar entre mujeres y que a veces crean situaciones que sólo una mujer puede comprender» ${ }^{35}$.

32 Este planteamiento sugiere que Rodrigo no era ajena al impulso que, sobre todo desde 1918, había adquirido en España el movimiento de las mujeres por la mejora de sus derechos sociales. FAGOAGA, C. (1985), La voz y el voto de las mujeres. El sufragismo en España 18771931, Barcelona, Icaria, pp. 123 y ss.

33 RodRigO, (1926a), p. 45. Subrayado en el original.

34 Esta posición ha sido indicada como una característica de las ideas progresistas sobre la educación de las niñas. La «reorientación del discurso de la desigualdad de las capacidades de los sexos, hacia otro más políticamente correcto de la diferencia entre los sexos», que se habría estimulado por higienistas y krausistas que sin embargo no cuestionaban el «estereotipo de `ángel del hogar` dominante, habría ayudado a «cristalizar el discurso dominante sobre las mujeres que siguió presionando para el inmovilismo en la construcción de la identidad femenina». FERNÁNDEZ VALENCIA, A. (2006), «La educación de las niñas: ideas, proyectos y realidades». En Morant, I. (ed.), Historia de las mujeres en España y América Latina. Volumen III. Del siglo XIX a los umbrales del XX, 427-453, Madrid, Cátedra, p. 446.

35 Rodrigo (1926a), p. 45. 
Ante la posibilidad de que esta última frase pudiera «herir la susceptibilidad masculina», la psicóloga del IRPIT expresaba su rechazo hacia «la idea de la superioridad de un sexo sobre otro» y manifestaba no compartir el punto de vista de «varias defensoras del movimiento feminista» que, «queriendo proteger su sexo contra juicios desfavorables», no encontrarían «nada más oportuno que reclamar para él todas las propiedades específicamente masculinas, incluso la dureza y el egoísmo ${ }^{36}$. De este modo, su categórica manifestación de que «los hombres y las mujeres son diferentes, es cierto», representaba un impulso para quienes estuvieran interesados en sostener una distribución de roles sociales basada en el sexo. Además, su intento de matizar esa afirmación con frases como que esas diferencias «no tienen que ser de valor, de cantidad», o que no se referirían «a un hombre o una mujer tomados individualmente, sino al tipo término medio», quedaban apagadas en medio de un escrito en el que, como veremos a continuación, se legitimaban esas diferencias y se tomaban como base para construir un discurso sobre la conveniencia de establecer, a través de la orientación profesional, «la manera de vivir y la ruta a seguir por la vida» de las mujeres ${ }^{37}$. En efecto, la autora que estamos siguiendo consideraba fundamental, para orientar a las niñas en su vida profesional, tener en cuenta la «enorme emotividad de las mujeres»; algo que según exponía representaba «un punto de la psicología sexual reconocido unánimemente ${ }^{38}$. Del mismo modo, y siguiendo ahora a Lombroso, afirmaba que «la inferioridad general de la mujer a la sensibilidad, a las excitaciones y a la sensibilidad diferencial», llevaban a la conclusión de que no sería oportu-

36 Rodrigo (1926a), p. 45. Creo que este planteamiento permite ubicar a Rodrigo en la posición teórica del feminismo que se ha dado en llamar «corriente dualista o relacional» y que fue la que prevaleció en España. Aunque ambas se oponían a la percepción social acerca de la inferioridad de las mujeres, la «corriente igualitaria» o «individualista» defendería la autonomía y se referiría a la persona con independencia del sexo o del género, mientras la «dualista» argüiría «la importancia de la facultad maternal de la mujer, facultad que no sólo tiene sus manifestaciones en el ámbito físico, sino en el psíquico y social». GóMEZ-FERRER, G (2006), «Introducción». En MorANT, I. (ed.), Historia de las mujeres en España y América Latina. Volumen IV. Del siglo XX a los umbrales del XXI, 13-27, Madrid, Cátedra, pp. 14-15.

37 RodRigo (1926a), p. 45. Mercedes Rodrigo se ubica así en un discurso científico sobre las mujeres que, como ha puesto de relieve Teresa Ortiz, había abandonado desde el inicio del siglo XX, especialmente en Medicina, un concepto basado en la inferioridad de las mismas con respecto al hombre para sustituirlo por otro cimentado en la diferencia. ORTIZ, T. (1993), «El discurso médico sobre las mujeres en la España del primer tercio del siglo XX». En LóPEZ BELTRÁn, M.T. (ed.), Las mujeres en Andalucía. II Encuentro Interdisciplinar de Estudios de la Mujer en Andalucía, 107-138, Málaga, Diputación Provincial, pp. 118-119.

38 RODRIGO (1926a), p. 45 
no emplear a las mujeres «en los trabajos en que es preciso distinguir diferencias muy pequeñas», como serían «la degustación de vinos, o de té, la afinación de pianos, etc.» ${ }^{39}$.

Mercedes Rodrigo establecía así un modo de aproximación para determinar las actitudes de los individuos para el trabajo en el que las diferencias en la constitución biológica y psicológica de los seres humanos se situaban en un lugar más que relevante ${ }^{40}$. No obstante, para ella, además de razones «de orden físico» y de orden «moral», había que tener también en cuenta factores de carácter «social» para llevar adelante la orientación profesional de las mujeres. La forma en que ponía de manifiesto la manera en que esos elementos debían ser objeto de atención en relación con la distribución de las tareas a desarrollar por hombres y mujeres limitaba así el alcance de su trabajo para romper con la tradicional distribución de roles. Es cierto que hablar de diferencia, en vez de inferioridad, a la hora de comparar al hombre y a la mujeres, propiciaba, como ha expresado Teresa Ortiz, una definición «biológica» de la mujer más compleja que facilitaba el reconocimiento de la diversidad de las mujeres y con ello «la posibilidad de salirse de un determinado rol comenzaba a reconocerse ${ }^{41}$, pero también lo es que el tipo de discurso que sostenía Mercedes Rodrigo contribuía a legitimar como algo natural una determinada función social para la mujer. En efecto, según exponía,

«En realidad toda mujer debería prepararse para dos profesiones: una que podríamos llamar del hogar para realizar conscientemente los deberes que impone la vida de familia y otra buscarla, de acuerdo con sus aptitudes en inclinaciones para el caso de que se quede solo para afrontar la vida, bien sea de soltera o de viuda.

(...) No negamos que la carrera normal de la mujer es el matrimonio y la maternidad. ¿Pero pueden o quieren seguirla todas? Convendrán conmigo en que no. Además, encuentran ustedes moral el que una muchacha pase toda su juventud sin otra ocupación que la caza del marido? ¿Y si no llegan a encontrar su media naranja?» ${ }^{42}$

Mercedes Rodrigo daba así por sentado que el destino «normal» de la mujer era el de ser esposa, madre y guardiana del hogar conyugal. La Orienta-

39 Rodrigo (1926a), p. 46

40 Teresa Ortiz ha destacado cómo esta nueva forma de contemplar a la mujer desde sus diferencias con respecto al hombre permitía «resolver las contradicciones del discurso social del momento, con numerosas mujeres participando en la esfera pública (...), ponía cotidianamente de manifiesto». ORTIZ (1993), p. 119.

41 Ortiz (1993), p. 119.

42 Rodrigo (1926a), p. 47. 
ción profesional femenina tenía una función de enfocar a las mujeres hacia la ocupación que mejor se adecuara a sus características corporales y psicológicas, pero esa misión tenía un alcance restringido ya que se aplicaba sólo a la etapa en que la mujer no había alcanzado el matrimonio o en caso de que permaneciera soltera o quedara viuda. El hogar era el lugar en el que la mujer desempeñaría «su papel esencial» para el conjunto social, y Rodrigo se encargaba de dejar explícitamente claro que la disciplina que cultivaba no debía ser contemplada como una amenaza para algo que era presentado como un principio de organización de la sociedad. Según lo expresaba, «la Orientación profesional femenina no [-pretendía-] desnaturalizar la función de la mujer» ${ }^{43}$, y esta función no sería otra, como estamos viendo, que la de ama de casa ${ }^{44}$.

De este modo, el discurso que se estaba desarrollando desde el IRPIT se mostraba limitado para acabar de transformar el modelo vigente en ese momento de distribución de roles sociales. El enfoque que se estaba desarrollando en él para aplicar los principios que se propugnaban desde la OCT contribuía a potenciar y consolidar la idea de la diferencia entre hombres y mujeres $\mathrm{y}$, a través de ello, a fortalecer una distribución del trabajo en la que el sexo representaba, al asumir que el destino natural de la mujer estaba en el hogar, un punto de referencia fundamental. Pero es que, además, cuando se trataba de plantear, desde las enseñanzas de la Orientación profesional, cuáles debían de ser las carreras o profesiones más recomendables para la mujer, la disciplina contribuía a enfatizar las diferencias entre ambos sexos. Las palabras de Rodrigo eran, en este sentido, significativas. A la hora de responder a esa cuestión manifestaba que:

\footnotetext{
43 Rodrigo (1926a), pp. 47-48.

44 Rodrigo parece instalarse así en línea con la posición que se ha designado como «el segundo paso en la evolución ideológica» sobre el tema de la actividad laboral femenina, que estaría ubicada entre la de oposición absoluta a la misma y la del «régimen de igualdad»al que se aspiraba. Según Capel, dicha posición, que desde mi punto de vista Rodrigo contribuía a fortalecer con su trabajo, no suponía «una amenaza económica ni sicológica al sistema establecido» por varias razones. La primera sería que sostenía que la participación de la mujer en las tareas productivas es considerada complementaria o sustitutiva de la masculina. El matrimonio era contemplado como la mejor manera de asegurarse el futuro, pero consideraba que la mujer debía adquirir la formación necesaria para ganarse su sustento en caso de quedar soltera o enviudar. La segunda era que la esfera profesional femenina debía venir determinada por el principio de «secundum naturam»; esto es, de acuerdo con las aptitudes individuales, pero sin romper «esta norma general que deja fuera de la gama de opciones todos aquellos puestos para los cuales carece de capacidad, la tiene muy limitada o resultan lesivos a la maternidad». CAPEL, M.R. (1986), El trabajo y la educación de la mujer en España (1900-1930), Madrid, Ministerio de Cultura. Instituto de la mujer, p.54.
} 
«Existen muy diversas opiniones. Hay quien cree que puede hacerlo todo y hay quien cree no puede hacer nada. En el terreno de las posibilidades puede decirse que actualmente toda profesión masculina es accesible a la mujer, pero no debe perderse de vista la conveniencia de que a pesar de la posibilidad teórica de ejercer cualquier posición, le conviene en interés propio y de la Sociedad, limitarse a algunas de ellas solamente, principalmente a aquellas en que, no sólo puede igualar al hombre, sino sobrepujarle» ${ }^{45}$.

La propuesta de organización del trabajo que se planteaba, en beneficio de cada mujer y de la sociedad en su conjunto, hacía por tanto hincapié en la idea de la diferencia, en el postulado de que cada uno de los sexos estaría más dotado para unas actividades que para otras. La consecuencia era que se podía establecer un catálogo de ocupaciones más adecuadas para la mujer -las que exigieran «actividad, intuición, pasión, rapidez de decisión, paciencia» y aquellas «relacionadas con la «emotividad y en las que se trate de hacer algo concreto»-, y otras que sería más conveniente reservar a los hombres -las que tuvieran relación con «lo abstracto y especulativo» y las que precisaran «reflexión, iniciativa» y se alejaran «de un objeto vivo y emotivo» ${ }^{46-}$. Tomando esto como base, la recomendación concreta para las mujeres a la hora de orientarlas hacia una tarea laboral era que eligieran «en primer lugar todas las profesiones relacionadas con los trabajos propios del hogar, cuidado de niños, incluso los trabajos agrícolas», el «cultivo de flores», el trabajo en hoteles, «las industrias del vestido» y las «carreras administrativas». Entre «las carreras liberales, las «preferibles para la mujer» serían aquellas que las ponía «en relación con la vida concreta y emotiva», entre las que se encontrarían:

«la enseñanza, porque en ella puede dar rienda suelta a su instinto maternal estando rodeada de seres indefensos, que constantemente necesitan sus cuidados; la medicina, que le permite encontrar el medio de procurar alegrías, de aliviar dolores, de aumentar la vida alrededor de ella; las carreras sociales, como directora de hogar para huérfanos, inspectora de trabajo, enfermera, etc., que exigen tanta penetración y comprensión, al mismo tiempo que una gran ternura; la música, la pintura, la literatura, y tantas otras» ${ }^{47}$

Este catálogo de ocupaciones que serían más adecuadas para la mujer coincidía con el que se elaboró a través de las aportaciones que fueron realizadas en el primer Congreso de Orientación Profesional Femenina, celebrado en

\footnotetext{
45 Rodrigo, (1926a), p. 48. El subrayado es mío.

46 Rodrigo, (1926a), p. 48.

47 Rodrigo (1926a), pp. 48-49.
} 
Burdeos con una presencia «en aplastante mayoría femenina» en septiembre de $1926^{48}$. La propia Mercedes Rodrigo informaba sobre el desarrollo del mismo y exponía cómo las carreras agrícolas, el servicio doméstico y las ocupaciones en los hoteles, en concreto las tareas de oficina, domésticas y vigilancia y administración interior, resultaban, como «las carreras de enseñanza», las «literarias» y las «sociales» (delegadas de protección de la infancia, empleadas de administración de la beneficencia, visitadoras sociales, inspección del trabajo o bibliotecarias), las más adecuadas para la mujer. Respecto a los «oficios manuales, de oficinas, comerciales y de arte aplicado» el Congreso pondría de manifiesto que los mejores para ellas

«son los que más respetan su naturaleza, ya sea porque las condiciones en que se ejecutan, la permiten llenar su misión en la familia, o porque sus exigencias están de acuerdo con las tendencias esencialmente femeninas.

Deberán, pues, escoger las profesiones que respondan mejor a las exigencias del 'temperamento femenino', en el que predomina la emotividad, el ingenio, la intuición; temperamento en el que la inteligencia aparece muy clara en los detalles pero que se mueve difícilmente en las ideas generales» ${ }^{49}$

De acuerdo con ello, la directora del Centro Femenino de Orientación Profesional de Burdeos habría clasificado en cuatro grupos los oficios manuales «propiamente femeninos: industrias del vestido, decoración del hogar, industria del libro y sus anejos y metalurgia y mecánica (especialmente relojería y fabricación de prótesis dentarias) $\rangle^{50}$.

El discurso que estamos examinando contribuía así a reafirmar la vieja idea de que la mujer tendría unas características corporales y psicológicas que condicionaban la eficacia con la que podían desarrollar su actividad laboral en determinadas ocupaciones. No obstante, conviene también poner de manifiesto que, como se puede deducir del propio trabajo de Mercedes Rodrigo que hemos comentado arriba, existía otro elemento que, aunque se planteaba como dependiente también de la propia constitución femenina, tenía que ver más estrictamente con lo que se consideraba que representaba su función social por excelencia: la maternidad, el cuidado de los niños y la gestión del hogar conyugal. En efecto, según se había puesto de relieve en el Congreso,

48 Rodrigo, M. (1926b), «Informaciones. Congreso Internacional de Orientación Profesional Femenina», Memorias del Instituto de Reeducación Profesional de Inválidos del Trabajo, 3, 99-103, p.99.

49 Rodrigo (1926b), p.101. El subrayado es mío.

50 RODRIGO (1926b), p.101. 
la persona encargada de realizar la Orientación social femenina se encontraría «en presencia de dos hechos al parecer incompatibles»: primero, «la necesidad de orientar a las muchachas teniendo en cuenta sus capacidades, sus gustos, su temperamento, con objeto de aumentar el rendimiento»; y segundo, «la necesidad de hacer de todas las mujeres, sea cual sea su competencia, buenas amas de casa» ${ }^{51}$. De este modo, la necesidad de tener en cuenta que la mujer estaría destinada a desempeñar ineludiblemente esta última tarea les llevaba por ejemplo a especificar cuáles serían, entre «las carreras de la higiene y de la medicina» las más apropiadas para ella. En ese sentido, las «carreras médicas propiamente dichas», como serían los «estudios médicos», la «práctica de la carrera de medicina, cirugía, especialidades» y la «dirección de sanatorios», no serían recomendables para la mujer, ya que sería «indispensable» para ejercerlas

«tener muy buena salud, dominio de sí misma, discreción, tacto y prudencia. La práctica de la medicina tiene más inconvenientes que ventajas para la mujer, por ser profesión fatigosa, que exige en ocasiones salir sola durante la noche y penetrar en todos los medios. Además, de no limitar su actividad a la consulta en su propio domicilio, la profesión médica es absolutamente incompatible con una actividad maternal normal y resulta demasiado absorbente y fatigosa para que pueda al mismo tiempo ocuparse la mujer de su hogar y el cuidado de sus hijos ${ }^{52}$

De este modo, como indicamos arriba ${ }^{53}$, los planteamientos de Mercedes Rodrigo se mostraban muy acordes con las teorías que caracterizaban eso que se ha denominado como «el segundo paso en la evolución ideológica» sobre el tema de la actividad laboral femenina, y que defendía el derecho de la mujer al trabajo socialmente productivo «siempre que se llevase a cabo en determinadas circunstancias - ausencia del esposo - dentro de unos límites - los impuestos por su 'naturaleza' - y controladas sus posibles consecuencias emancipadoras $\rangle^{54}$. Sus puntos de vista contribuían a dar aliento a esta posición, pero facilitaban poco la aceptación y difusión de otra postura, «defensora de una igualdad legal y real de ambos sexos en los distintos aspectos de su actividad productiva», que también se hallaba presente en la España anterior a 193055. Considero

\footnotetext{
51 RODRIGO (1926b), p.101.

52 RodRigo (1926b), p. 102.

53 Véase lo indicado en la nota 44.

54 CAPEL (1986), p. 55.

55 CAPEL (1986), pp. 55-56 ha señalado que esta posición, mantenida por las ideologías revolucionarias, tuvo una escasa difusión, y atribuye ésta a «las vicisitudes por las cuales pasa
} 
que Rodrigo se hallaba en dificultades para dar ese salto cualitativo que suponía romper con determinados prejuicios sobre las capacidades de la mujer en relación con la actividad laboral no sólo por los condicionantes sociales del momento, sino por el peso que sobre ella ejercían las ideas al respecto que, arropadas con el palio de la Ciencia, estaba generando el discurso de la OCT en que se había formado y que ella contribuía a difundir y desarrollar.

Aunque este artículo de Mercedes Rodrigo que acabamos de examinar es el único que hemos encontrado en que de manera monográfica se trata el tema de la orientación profesional de la mujer entre los producidos por quienes cultivaban la OCT en España, es posible encontrar datos en algunas otras de las aportaciones teóricas y de las actividades prácticas de quienes cultivaban la disciplina para avalar cómo desde esas posiciones se defendía una distribución social del trabajo en la que el sexo había de ser considerado como un parámetro de primer orden.

UNA NUEVA TECNOLOGÍA PARA LA DISTRIBUCIÓN «RACIONAL» DE LOS TRABAJADORES

Como estamos viendo, la creciente, y ya cuantitativamente relevante, contribución de la mujer al trabajo remunerado ${ }^{56}$, se pretendía someter a la acción de ese tamiz que representaba la Orientación profesional. Desde esta disciplina se mantenía un discurso respecto a la incorporación de las mujeres al mercado laboral en el que sus características corporales y psicológicas, y la idea de que su «carrera normal» era el matrimonio y la maternidad, representaban elementos fundamentales a tener en cuenta de cara a llevar a cabo una distribución adecuada y rentable del mercado laboral asalariado.

La Medicina y la Psicología encontraban así, a través de la Orientación profesional, un fértil campo de trabajo para desarrollarse. No debe extrañar

su historia en la España anterior a 1930, unidas al limitado potencial numérico de obreras industriales y al conservadurismo que impregna por igual a todas las capas sociales», que habrían amortiguado «su eco limitándolo a ciertos sectores laborales incapaces de generar un movimiento más amplio a favor de los ideales igualitarios que, por otra parte, sólo son expuestos de forma esporádica en algunos artículos»

56 Mercedes Rodrigo estimaba que la mujer se vería «cada día más inclinada» al trabajo profesional, «no por motivos de emancipación, como se cree con demasiada frecuencia, sino a causa de la carestía de la vida, del incesante desarrollo de la vida económica e intelectual, que ha hecho absolutamente necesario el concurso de la mujer en la industria, en los oficios, en los comercios y en otras manifestaciones de la vida». RODRIGO (1926a), p. 49. 
que, quienes trataban de legitimar la nueva disciplina, y de mostrar su relevancia para la buena marcha de la sociedad, se afanaran en hacer hincapié en las diferentes características psíquicas y somáticas de los individuos aunque ello pasara por reproducir y justificar, eso sí, rodeándolo de la deslumbrante aureola de la Ciencia, la tradicional distribución de roles entre hombres y mujeres. En línea con los objetivos de la OCT de racionalización de la actividad laboral se imponía ubicar a cada individuo en el lugar de trabajo que mejor correspondiera a sus características psíquicas y somáticas, no sólo para hacerlo más productivo, sino más feliz. Interés social e interés individual justificaban que la Orientación profesional se desarrollara y que el Estado hiciera un esfuerzo por impulsar su institucionalización. Como se ponía de relieve en un congreso dedicado a esa disciplina en 1926 en Vitoria, el concurso de la misma se hacía necesario «al conjuro de tantos accidentes del trabajo, carreras fracasadas y crisis de producción $\rangle^{57}$. Como veremos a continuación esa era la línea en la que se estaba trabajando ya en el IRPIT y que estaba sirviendo para poner a punto una tecnología médico-psicológica de la que, como acabamos de ver, se derivaba una distribución sexual de la actividad laboral ${ }^{58}$.

Desde el principio estuvo claro que el IRPIT había sido concebido para cubrir una función que iba más allá de la de capacitar a las víctimas de los accidentes del trabajo para reintegrarse en la actividad laboral. Como comentaba uno de los miembros del Patronato que habría de regir su creación, entre las razones que habían servido para justificar su implantación habrían figurado también: evitar la caridad, disminuir el «quebranto» que producirían en la base de la riqueza pública los accidentes del trabajo, y acortar los períodos de invalidez ${ }^{59}$. De este modo, el programa de actuaciones que se planteaban desde el Instituto pretendía también racionalizar la vida económica de la nación y contribuir a mejorar la organización del trabajo en España. En ese sentido, la

57 «Informaciones. Concurso de Orientación profesional en Vitoria», (1926), Memorias del Instituto de Reeducación Profesional de Inválidos del Trabajo, 3, 104.

58 Utilizo tecnología médico-psicológica en el sentido en que el concepto de tecnología médica ha visto redimensionado su significado desde la última década del pasado siglo. Frente a la simple alusión a las «máquinas», se ha ido incluyendo todo aquello que se diseña para entender, prevenir, diagnosticar y tratar la enfermedad y los desórdenes físicos y corporales. Brown, N.; Webster, A. (2004), New Medical Technologies and Society. Reordering the Life, Cambridge, Polity Press, p. 4; MEnÉndeZ, A.; MedinA, R. (2004), «Tecnologías médicas en el mundo contemporáneo: una visión histórica desde las periferias. Introducción», Dynamis, 24, 15-26, p. 16.

59 DeCREF, J. (1924), «La reeducación de inválidos para el trabajo», El Siglo Médico, 73, 414-416, 445-447; 468-470, pp. 414-415. 
disminución de los daños que producía en la economía y en los trabajadores la presencia de altas tasas de siniestralidad representaba, por su interés general, un objetivo apropiado para el centro ${ }^{60}$. De hecho, se hizo un esfuerzo importante desde él con vistas a alcanzarlo ${ }^{61}$. Se recurrió para ello a los medios que la OCT proponía, y de forma destacada a la orientación y la selección profesionales $^{62}$.

La tecnología médico-psicológica preventiva que se impulsó desde el Instituto para combatir la siniestralidad laboral partía de la búsqueda de una fórmula que fuera capaz de «reducir el número de accidentes sin disminuir la producción ${ }^{63}$, y que contara con el apoyo de patronos y trabajadores. La propuesta que realizaron tenía como ingrediente fundamental su insistencia en poner de relieve los factores dependientes del trabajador en la etiología del accidente. Se trataba de destacar la responsabilidad del obrero en su producción, de pasar a primer plano la «culpabilidad consciente o inconsciente» del que «provoca el accidente» ${ }^{64}$, y de situar los factores dependientes de las malas condiciones del medio laboral en un lugar secundario. Dado que, según defendían, «las condiciones psico-fisiológicas de los individuos son las que deciden principalmente la suerte del trabajador ante los peligros del traba-

60 Según se ponía de relieve en esa época, la prevención de la siniestralidad laboral debía interesar «a los patronos», al ocasionar «un gasto en la producción» y representar «una carga por su coste y por las horas perdidas de trabajo»; pero, asimismo, habría de interesar a los obreros por servir a «la conservación de su salud» y a combatir «la merma» que producirían los accidentes en sus ingresos. De ANDRÉs BuENO, V. (1934): La prevención de los accidentes del trabajo, Valladolid, Cuesta, p. 2.

61 Me he ocupado del modelo desarrollado en el IRPIT para la prevención de la siniestralidad laboral en: MARTínEZ-PÉREZ, J. (1994), «La Organización Científica del Trabajo y las estrategias médicas de seguridad laboral en España (1922-1936)», Dynamis, 14, 131-158; y (2001), «Medicina del Trabajo y prevención de la siniestralidad laboral en España (19221936)», en AtEnZA, J.; MARTíNEZ-PÉreZ, J. (eds.), El Centro Secundario de Higiene Rural de Talavera de la Reina y la Sanidad Española de su tiempo, Toledo, Junta de Comunidades de Castilla-La Mancha, 235-257.

62 Mallart, por ejemplo, afirmaba que entre las medidas destinadas a evitar los accidentes debían figurar: «desviar de las ocupaciones de peligro a los que por sus dolencias son candidatos al accidente (Orientación profesional médica)», y «colocar a cada individuo en el trabajo que esté más adecuado con sus aptitudes naturales y con sus defectos (Orientación profesional fisiológica y psicotécnica, selección profesional)». MALLART, J. (1926) «Orientación profesional y prevención de accidentes del trabajo», Memorias del Instituto de Reeducación Profesional de Inválidos del Trabajo, 3, 50-61, p. 57.

63 MALLART (1926), p. 51.

64 MaLlart (1926), p. 53. 
jo» ${ }^{65}$, la intervención sobre el «factor humano» se convertía así en el objetivo prioritario que se proponía desde el IRPIT para combatir la siniestralidad laboral. Mercedes Rodrigo sostenía que «la mayor parte de las personas» que habían estudiado el problema de la prevención de los accidentes del trabajo estarían «de acuerdo en conceder enorme importancia al factor constitucional y reconocer la necesidad absoluta de estudiar la personalidad del obrero si se quiere obtener resultados eficaces» a ese respecto; y añadía que «el exacto conocimiento del individuo puede explicar la razón por la cual se produce la causa violenta origen del accidente» ${ }^{66}$. De este modo, como indicaba otro de los psicopedagogos del centro,

«Las aptitudes naturales, los defectos de constitución, la enfermedad o aun la predisposición para la enfermedad, han de ser objeto de una atención escrupulosa al determinarse la entrada de un aprendiz en una industria o al decidir la vía profesional, que ha de seguir un joven. Se ofrecen muchos trabajos en los cuales hay poco peligro; pero desgraciado el individuo y desgraciada la empresa si ocupa a un aprendiz en una vía de trabajos que no están en consonancia con las defensas individuales que habrían de anular o de reducir a un mínimo despreciable el riesgo del trabajo, máxime cuando estas defensas están íntimamente ligadas, como parecen estar en la mayoría de los casos, con las aptitudes naturales que requiere la ejecución del trabajo desde el punto de vista del rendimiento» ${ }^{67}$

Considerado, por tanto, el trabajador como uno de los factores fundamentales en la etiología de los accidentes del trabajo, y siendo uno de los objetivos a alcanzar su mejor rendimiento, se hacía necesario poner en marcha una serie de estrategias encaminadas a conseguir lo uno y lo otro ${ }^{68}$. La formación, la orientación y la selección profesionales, que el IRPIT estaba contribuyendo a desarrollar, acudían como disciplinas adecuadas para cubrir esa función.

Estos planteamientos encontraron continuidad en el Instituto Psicotécnico de Madrid (IPM, en adelante $)^{69}$, entre cuyas funciones principales figuraban:

65 MALlart (1926), p. 55.

66 Rodrigo, M. (1929), «La prevención de los accidentes del trabajo». En Oller, A. et. al., La práctica médica de los accidentes del trabajo, Madrid, Javier Morata, 388-415, p. 392.

67 Mallart, J. (1932b): «La prevención de los accidentes del trabajo en la industria», Medicina del Trabajo e Higiene Industrial, 3, 34-49, p. 43.

68 La OCT se erigía en efecto, según lo ha expresado Sierra Álvarez, como un programa de técnicas que debían aplicarse «en la fábrica y fuera de ella, sobre el trabajo y sobre el trabajador» para conseguir el tipo ideal de éste que exigía la industria». SIERRA ÁlvAREZ (1990), p. 28.

69 Sobre el desarrollo del Instituto pueden verse, además de las obras citadas en la nota 
«a) La investigación de los problemas del factor humano en el trabajo; el estudio de las aplicaciones de la fisiología y de la psicología humanas en todos los sectores de la actividad donde puedan introducir mejoras de rendimiento, y la aplicación misma de los principios de la ciencia del trabajo en la organización de los servicios públicos y de las empresas industriales, mercantiles y agrícolas de todo orden.

b) El estudio y la organización de la orientación profesional y de la selección profesional en su más amplio sentido.

c) La elaboración, tipificación y establecimiento de tests y de técnicas psicológicas para estudio y clasificación de los individuos para fines pedagógicos, profesionales, sociales, etc.» ${ }^{70}$.

Se trataba, pues, de tener en cuenta la diversidad humana a la hora de racionalizar la vida laboral, de estudiar y clasificar a los individuos de acuerdo con sus características somáticas y psicológicas para que desarrollaran con más eficacia y seguridad su trabajo. Fueron esas las razones por las que una de las cuestiones que interesó también desde un primer momento fue la del modo en que esos rasgos de los individuos se relacionaban con el grado de resistencia a la fatiga ${ }^{71}$. De este modo, el discurso que estamos analizando contenía por tanto ingredientes que, al hacer hincapié sobre las diferencias entre las personas, facilitaba la defensa de una distribución del trabajo entre cuyos criterios figurara de forma destacada la categoría «sexo».

29: Mallart, J. (1933), «La Orientación profesional en España», Medicina del Trabajo e Higiene Industrial, 4, 33-92, 40-43; y EnCINA; Rosa (1990), pp. 84-87.

70 MaLLART (1932a), p. 107-108.

71 Antonio Melián, que fue miembro del IRPIT y Jefe del Departamento médico del IPM, llamaba la atención sobre su relación directa con la disminución del rendimiento y con la aparición de la siniestralidad laboral. MeLián, A. (1927), «La fatiga industrial (Estado actual del problema)», Memorias del Instituto de Reeeducación de Inválidos del Trabajo, 4, 39-72, pp. 66, 68. También José Germain, el Director del IPM, indicaba que la aparición de la fatiga determinaría que el organismo quedara «a merced de lo que le rodea. Ese sería el momento de los accidentes en una gran mayoría de casos» GERMAIN, J. (1932), «La fatiga en la industria», Medicina del Trabajo e Higiene Industrial, 3, 131-148, 379-390, pp. 141-142. De este modo, la investigación en torno a la fatiga servía a dos fines: «1. Organizar el trabajo en forma que se obtenga el máximo rendimiento con el mínimo esfuerzo (racionalización); 2 . Servir de base a la orientación y selección profesional». DANTín, J. (1935), «La fatiga y su medida. Capacidad física», Revista de Organización Científica, 4, 695-715, p. 707. Acerca del uso del concepto de fatiga para el desarrollo de la Medicina del Trabajo en España puede verse: MARTínEZPÉREZ, J. (1998), «La fatiga industrial: un concepto estratégico en el desarrollo de la Medicina y Psicología del Trabajo en España (1927-1936)». En CASTELlanos, J. et al. (eds.), La Medicina en el Siglo XX. Estudios históricos sobre Medicina, Sociedad y Estado, Málaga, Sociedad Española de Historia de la Medicina, 133-145. 
Como he tratado de poner de manifiesto, la consideración de que el estudio del «factor humano» resultaba de capital importancia para una mejor y más científica organización del trabajo justificaba la puesta en marcha de toda una tecnología médica y psicológica - cognitiva, técnica e institucional- destinada a asignar a las personas diferentes roles dentro de la actividad laboral. Aunque en el caso de la fatiga las exploraciones destinadas a medirla no eran consideradas todavía eficaces ${ }^{72}$, este hecho estaba lejos de desanimar a quienes consideraban que el examen de las características corporales y mentales de los individuos era un elemento fundamental para llevar a cabo una buena orientación y selección profesionales. En efecto, el «examen médicoantropométrico»y el «psicotécnico» de los individuos se convirtió en un requisito imprescindible dentro del proceso establecido por el IPM para determinar qué profesión resultaba «funcionalmente» conveniente a una persona y encajaba mejor con sus aptitudes ${ }^{73}$.

Y es que buena parte de la justificación de esas actuaciones tenía que ver con el importante grado de seguimiento y convicción que los médicos y psicólogos de ese centro tenían hacia la Patología constitucional ${ }^{74}$. En relación, por ejemplo, con los accidentes del trabajo, estimaban que la constitución ${ }^{75}$,

«por su repercusión sobre los cambios nutritivos, sobre el tono de las funciones neurovegetativas, así como sobre el tipo hormónico (sic) dominante en el individuo - por una parte - y por otra parte el temperamento psíquico, tienen una considerable influencia sobre las causas y evolución de los accidentes» ${ }^{76}$

72 Germain, por ejemplo, manifestaba que, «en el estado actual de la ciencia, nuestro conocimiento de la fatiga no nos permite poderla medir». GERMAIN, J. (1932), «La fatiga en la industria», Medicina del Trabajo e Higiene Industrial, 3, 131-148, 379-390, p. 390.

73 Mallart (1932a), p. 110

74. Una visión de conjunto sobre el desarrollo del pensamiento médico en torno a la influencia de la constitución somática en la aparición de los problemas de enfermedad puede verse en: OlBY, R.C. (1993), "Constitutional and Hereditary Disorders». En BYNUM, W.F.; PORTER, R. (eds.), Companion Encyclopedia of the History of Medicine (2 vols.), London, Routledge, 1, 412-437.

75 Como ha expuesto Laín, los clínicos de la época empleaban el término «constitución» para hacer alusión a una «disposición» o a una «resistencia» respecto a una determinada enfermedad, pensando que una y otra tiene una estructura biológica que se hallaría configurada por un componente hereditario y otro adquirido. LAÍn ENTRALGO, P. (1961), La historia clínica. Historia y teoría del relato patográfico (2 edición), Barcelona, Salvat, p. 400.

76 GRANDA, B. (1929), «Constitución y accidente del trabajo, Reeducación profesional», Memorias del Instituto de Reeducación Profesional de Inválidos del Trabajo, 5-6, 11-21, p. 19. 
Así, pues, según defendían, la estructura biológica no sólo influiría sobre las funciones digestivas, nerviosas y hormonales, sino sobre el perfil psicológico de las personas. Muy explícito era a este respecto Antonio Melián, al afirmar que «el concepto de constitución» comprendería «el modo de reaccionar a los estímulos normales y patológicos que se definen generalmente aparte, con el nombre de temperamento $\rangle^{77}$. No puede extrañarnos, por tanto, que su respuesta a la pregunta sobre si existiría una correlación entre los rasgos físicos del hombre y su carácter no fuera otra que la «afirmativa» ${ }^{78}$. Tampoco puede sorprendernos que, al establecer las causas de la fatiga, y al señalar aquellas que «guardan relación con las condiciones psico-fisiológicas del individuo», situara entre ellas al «sexo» ${ }^{79}$.

De este modo, las diferencias sexuales se convertían en un elemento a tener en cuenta a la hora de llevar a cabo una tarea, tan determinante de cara a la configuración de una organización social de género, como era la de distribuir a las personas en las diferentes actividades laborales. En ese sentido, Antonio Oller manifestaba que

«en la organización del trabajo tiene una importancia capital la resistencia del obrero, ya porque se trate de sujetos débiles, mujeres y niños, o de sujetos tarados por alguna enfermedad anterior, o simplemente de sujetos que no reúnan las aptitudes necesarias para el oficio a que se dedican o se cansen más pronto y con más intensidad que los normales» 80

La mujer se presentaba así situada al lado de los «débiles», de los niños y de aquellos varones que se cansan antes y más intensamente que los «normales». La mujer se seguía calibrando, como en etapas anteriores, frente al prototipo que representa el varón sano ${ }^{81}$. Respecto al tema que ahora nos incumbe, esto representaba en la práctica descartar a las mujeres para todo un rango de oficios y tareas por no poseer la fuerza necesaria, por su supuesta debilidad.

77 Melián, A. (1934), «Problemas médicos de la orientación y la selección profesionales», Revista de Organización Científica, 4, 363-381, p. 373.

78 MELIÁN (1934), p. 377.

79 Melián, (1927), p. 44.

80 Oller, A. (1934), Medicina del Trabajo, Madrid, Javier Morata, p. 167.

81 Catherine Jagoe ha indicado cómo en el siglo XIX español la salud tenía «un género, el masculino. El varón es la pauta del cuerpo sano, desde la cual se mide al sexo femenino». Jagoe, C. (1998), «Sexo y género en la Medicina del siglo XIX». En JagOE, C.; BlanCo A; ENRÍQueZ De SAlamancA, C. (eds.), La mujer en los discursos de género. Textos y contextos en el siglo XIX, Barcelona, Icaria, pp. 305-367.p. 307. Véase también lo indicado en nota 10. 
Por consiguiente, desde el discurso que estamos examinando se estaba contribuyendo a fortalecer determinados estereotipos que la Medicina había contribuido a establecer acerca de las mujeres. No obstante, respecto al tipo de actividades laborales que resultaban más adecuadas para la mujer en función de su constitución, los autores aquí estudiados se mantenían reservados y se expresaban de un modo más implícito que explícito. Esta situación pudo deberse a varias razones. Junto al hecho de que el tema podía despertar un cierto grado de polémica ${ }^{82}$, cabe pensar también, como he indicado en otro lugar, en el influjo de factores relacionados con intereses profesionales y con el grado de desarrollo del saber y la práctica de la orientación profesional ${ }^{83}$.

A pesar de lo anterior, lo cierto es que en los documentos que generaban sí se expresaba de forma manifiesta el sesgo de género que incorporaba el discurso que estamos explorando. De hecho, las fichas «fisiológica», «psicológica» y «psicotécnica» que se cumplimentaban en el Laboratorio de Orientación profesional del IRPIT poseían un espacio para marcar si la persona que había de ser reeducada era hombre o mujer ${ }^{84}$. Igualmente, en el «cuestionario psicofisiológico de las profesiones», que los miembros del IPM elaboraron con el objetivo de conocerlas y de proceder a orientar a un individuo hacia la que se consideraba más apropiada para él, contenía preguntas sobre el «sexo más adecuado» para ejercerlas y sobre si la profesión en cuestión se podía considerar «privativa de un solo sexo» ${ }^{85}$. Como los cuestionarios eran cumplimentados por quienes estaban ejecutando cada uno de los tipos concretos de actividad laboral para los que se deseaba evaluar la aptitud de un individuo $^{86}$, los prejuicios sociales hacia las capacidades de la mujer para determinadas tareas, tanto para las que les estarían vedadas como para las que les eran más propicias, podían incorporarse a la hora de establecer las condiciones requeridas para ejecutarlas. De este modo, los médicos y psicopedagogos del IRPIT y del IPM, al tomar estas fichas como referencia en su labor de

82 Sobre esta cuestión puede examinarse NASH, M. (1981), La mujer y el movimiento obrero en España, Barcelona, Fontamara. El reflejo de esas polémicas en relación con la Medicina ha sido señalado por ORTIZ, T. (1993).

83 MARTínEZ-PÉREZ, J. (2004), «Diversidad humana y rendimiento laboral: sobre accidentes de trabajo, Medicina y organización social de género en España (1922-1936)». En MARTÍNEZ-PÉREZ, J. et. al. (eds.), La Medicina ante el nuevo Milenio: una perspectiva histórica, Cuenca, Ediciones de la Universidad de Castilla-La Mancha, 83-102, pp. 93-97.

84 OLLER (1924), pp. 159,163,166.

85 MALlaRT, J. (1932d), «El estudio de los trabajos para su organización científica y para la orientación profesional», Medicina del Trabajo e Higiene Industrial, 3, 399-417, p. 402.

86 MALlaRT (1932d), pp. 400-401. 
orientación profesional, contribuían así a fortalecer una organización social de género, a legitimar con su discurso y sus prácticas científicas la distribución sexual de los puestos de trabajo vigente en España.

\section{A MODO DE EPÍLOGO}

No me es posible establecer el grado de seguimiento que se hizo en España de los planteamientos de la OCT sobre el modo más conveniente de distribuir a las personas para desempeñar un trabajo remunerado. Como reconocía una de sus principales impulsoras, el desarrollo del discurso era aún incipiente en esa etapa como para que sus contenidos teóricos y prácticos se plasmaran con rotundidad en la realidad del mercado laboral ${ }^{87}$. No obstante, es posible indicar que a comienzos de la década de 1930 las ocupaciones que desempeñaban con mayor frecuencia las mujeres eran precisamente aquellas que la OCT señalaba como más adecuadas a sus características corporales y psicológicas $^{88}$. De hecho, el discurso que estamos explorando, no sólo estuvo lejos de ser abandonado a consecuencia de la Guerra Civil, sino que aparecía, al menos en lo que respecta a la confianza en lo que podía ofrecer para la organización de la actividad laboral en España, robustecido. El estudio del «factor humano», y su aplicación para la distribución adecuada de los trabajadores en el mercado laboral, eran considerados «principios fundamentales» para ello y,

87 Rodrigo (1933), «Algunos problemas de orientación profesional», Medicina del Trabajo e Higiene Industrial, 4, 93-160, p. 158.

88 Mary Nash ha señalado que hacia 1930 las mujeres representaban el 12,5\% del total de la población activa y el $9,66 \%$ de su sexo. La distribución de las mujeres activas en los diferentes sectores económicos denotaba una mayor presencia de la mujer en la industria, el servicio doméstico y la agricultura, por ese orden. Pero, como indica la autora, la creciente participación de la mujer en la industria no implicaba la integración de la mujer en todas las ramas productivas, sino que, al contrario, «encontramos una mayor presencia suya precisamente en estas industrias de transformación consideradas como más apropiadas a la naturaleza femenina»: la del vestido-tocado, y la textil. Esta distribución de la mano de obra femenina respondería para Nash, entre otras razones, a que la teoría de la división de las esferas negaba la capacidad y aptitud laboral de la mujer, o la consideraba inferior a la del varón. Cuando esta ideología se hubo de resignar a la incorporación de la mujer al proceso productivo, lo previó en aquellas áreas que se estimaban más adecuadas a su sexo, esto es, a las más «afines a las tareas desempeñadas en el hogar». Junto a esto, otra razón tuvo que ver con la convicción de que las mujeres poseían «un bajo nivel de rendimiento laboral y su absentismo laboral debido a la maternidad». NASH, M. (1983), Mujer, familia y trabajo en España, 1875-1936, Barcelona, Anthropos, pp. 50-52. 
según se sostenía, habrían «conquistado en las modernas tendencias de la industria mundial categoría de axioma» ${ }^{89}$.

En consonancia con estos planteamientos, las actuaciones encaminadas a lograr mejorar los problemas relacionados con la seguridad en el trabajo se iban a fundamentar en unos principios rectores que nos resultan familiares por haber sido ya formulados con anterioridad a la Guerra Civil: la idea de que el «factor humano» era una causa fundamental de siniestralidad laboral; la convicción de la existencia de una «predisposición individual al accidente», la necesidad de establecer las condiciones que debía poseer un obrero para realizar una determinada tarea laboral, y por fin, en consecuencia con lo anterior, el estudio de sus características anatómicas, fisiológicas y psicológicas ${ }^{90}$. No obstante, una diferencia era significativa. Me refiero al modo más abierto en que realizaban sus consideraciones de cara a establecer, basándose en esos principios, una organización de la actividad laboral en la que el sexo figurara como una categoría fundamental para realizarla. En efecto, la Psicotecnia, con sus aportaciones a la organización y racionalización del trabajo, debía de ser la base rectora de todas las decisiones de los organismos oficiales conducentes a regular las actividades relacionadas con el trabajo. En relación con esto, se indicaba explícitamente que la Psicotecnia imponía «limitaciones de edad y sexo para ciertas actividades, que resultarían eminentemente perjudiciales para el obrero» ${ }^{91}$.

De este modo, el discurso que, sobre la base de los principios teóricos de la $O C T$, se promovió desde mediados del primer tercio del siglo XX, se iba a adentrar también en el segundo. Es más, en relación con la manera en que, quienes lo sostenían y aplicaban, consideraban al sexo como un factor a tener en cuenta a la hora de proceder a la organización de la actividad laboral remunerada, como un criterio para establecer una lista de ocupaciones propias de hombres y otra de trabajos más adecuados para las mujeres, es posible apreciar que sus manifestaciones eran más explícitas que en la fase anterior a la Guerra Civil.

Fecha recepción: 2 de mayo de 2007

Fecha aceptación: 20 de junio de 2007

89 Cfr. Congreso Nacional de Medicina y Seguridad del Trabajo. Bilbao, 16-21 de agosto de 1943, (1944), Madrid, Ministerio de Trabajo, p. 31.

90 Ibarrola Monasterio, R. (1944), «La Psicotecnia del sujeto en relación con la prevención de accidentes y enfermedades profesionales». En Congreso (1944), pp. 207-292.

91 BORRÁs (1944), «La Psicotecnia del objeto en relación con la prevención de accidentes e higiene (Fundamentos y antecedentes para una ordenación psicotécnica industrial integral)». En Congreso (1944), 293-322, pp. 315 y 321. 